\title{
Waveguiding in planar photonic crystals
}

Marko Loncar, Dusan Nedeljkovic, Theodor Doll, Jelena Vuckovic, Axel Scherer, et al.

Marko Loncar, Dusan Nedeljkovic, Theodor Doll, Jelena Vuckovic, Axel Scherer, Thomas P. Pearsall, "Waveguiding in planar photonic crystals," Proc. SPIE 4293, Silicon-based and Hybrid Optoelectronics III, (18 May 2001); doi: $10.1117 / 12.426924$

SPIE. Event: Symposium on Integrated Optics, 2001, San Jose, CA, United States 


\title{
Waveguiding in Planar Photonic Crystals Marko Lončar ${ }^{a}$, Dušan Nedeljković ${ }^{b}$, Theodor Doll ${ }^{a}$, Jelena Vučković ${ }^{a}$, Axel Scherer ${ }^{a}$, and Thomas P. Pearsall $^{b}$
}

\author{
${ }^{\circ}$ Department of Electrical Engineering, Caltech, Pasadena, California 91125, USA \\ *Centre Européen de Recherche de Fontainebleau, Corning, SA 77210 Avon, France
}

\begin{abstract}
Photonic crystal planar circuits designed and fabricated in silicon on silicon dioxide are demonstrated. Our structures are based on two-dimensional confinement by photonic crystals in the plane of propagation, and total internal reflection to achieve confinement in the third dimension. These circuits are shown to guide light at $1550 \mathrm{~nm}$ around sharp corners where the radius of curvature is similar to the wavelength of light.
\end{abstract}

Keywords: Photonic bandgap, waveguide, silicon, index contrast

\section{INTRODUCTION AND BACKGROUND}

The study of integrated optics is more than 30 years old. The planar photonic crystal concept is a recent innovation ${ }^{1}$ that for the first time can permit the miniaturization of integrated optical circuits to a scale comparable to the wavelength of light. In principle, this new technology makes it possible to fabricate planar optical circuits with a packing density four to five orders of magnitude higher than the present state of the art, thus realizing for the first time the original objective of integrated optics.

Waveguiding in a planar circuit requires confinement of the light in three dimensions. This can be achieved by making a three-dimensional photonic crystal ${ }^{2-4}$. However, fabrication of good quality 3D photonic crystal structures is difficult process, and in terms of fabrication, a more appealing approach is based on the use of the lower dimensional photonic crystals to achieve confinement of light in all three dimensions. That idea is employed in the case of the thin dielectric slab perforated with two-dimensional photonic crystal ${ }^{5-10}$. In the vertical direction light is confined to the slab due to the Total Internal Reflection (TIR), and in the lateral direction is controlled by the means of Distributed Bragg Reflection (DBR) due to the presence of 2D photonic crystal. The photonic crystal structure that we consider here consists of a periodic arrangement of holes etched in a planar silicon slab suspended so that it is surrounded by air on both sides, processed using siliconcompatible fabrication technology. We have studied the propagation of light in this structure using methods based on plane wave expansion and finite-difference time domain to analyze Maxwell's equations ${ }^{5-10}$. In agreement with Johnson et al. ${ }^{11}$ we find that conditions for waveguiding are more restrictive in a waveguide with a finite $3^{\text {rd }}$ dimension than in a 2-dimensional photonic crystal of infinite extent in the third dimension ${ }^{12,13}$. Kuchinsky et al. ${ }^{14}$ have shown that a photonic band gap can exist in a planar waveguide structure only when its thickness is less than the period of the waveguide. Without a photonic band gap there can be no confinement, and no guiding of light.

The demonstration of waveguiding by photonic crystal confinement is a difficult challenge, but a necessary achievement in order to realize the goal of planar optical circuits with higher levels of integration. A recent paper by Tokushima et al. ${ }^{15}$ reports experiments on light propagation in a structure that lies well outside the parameter space where guiding can occur. Baba et al. ${ }^{16}$ reported experiments in structures of the appropriate design but with significant scattering losses. In this letter we demonstrate waveguiding of a well-defined mode at $1550 \mathrm{~nm}$ over hundreds of wavelengths of distance with waveguide losses below the detection threshold of our apparatus.

The dispersion relationship, that is, the photonic bandstructure, is the key element to understand the nature of equilibrium modes that can support low-loss waveguiding. In our previous publication ${ }^{8}$ we have presented the bandstructure calculations for silicon slab perforated with lattices of both square and hexagonal symmetry. We found that in both structures bandgap is opened for TE-like modes, around normalized frequency $\frac{a}{\lambda_{0}}=\frac{\omega a}{2 \pi c} \approx 0.35$ ( $a$ - the periodicity of the lattice, $\lambda_{0}-$ the wavelength in the air). When we introduce a line defect into the photonic crystal by removing an entire line of holes from the two-dimensional crystal lattice we form the simplest photonic crystal waveguide. Photons having an energy within the gap can 
now propagate only along this line defect. Propagation in the lateral direction is suppressed by the fact that the defect mode is within the frequency bandgap of the photonic crystal, and free-space propagation in the vertical direction is prevented by means of Total Internal Reflection. Three-dimensional analysis of this structure shows that straight-line defect, in the lattice of both hexagonal and square symmetry, can support guided modes around normalized frequency $\frac{a}{\lambda_{0}} \approx 0.35^{8,14}$.

\section{DESIGN AND NANOFABRICATION}

Waveguides were fabricated in a silicon on insulator (SOI) wafer, as single crystal silicon is known to be transparent within the interesting 1300 to $1700 \mathrm{~nm}$ wavelength range. Patterns are defined in PMMA, the only lithography mask used, using electron beam lithography and then transferred into silicon using Chemically Assisted Ion Beam Etching (CAIBE). The structure of the wafer and the whole fabrication procedure is shown in the Figure 1.

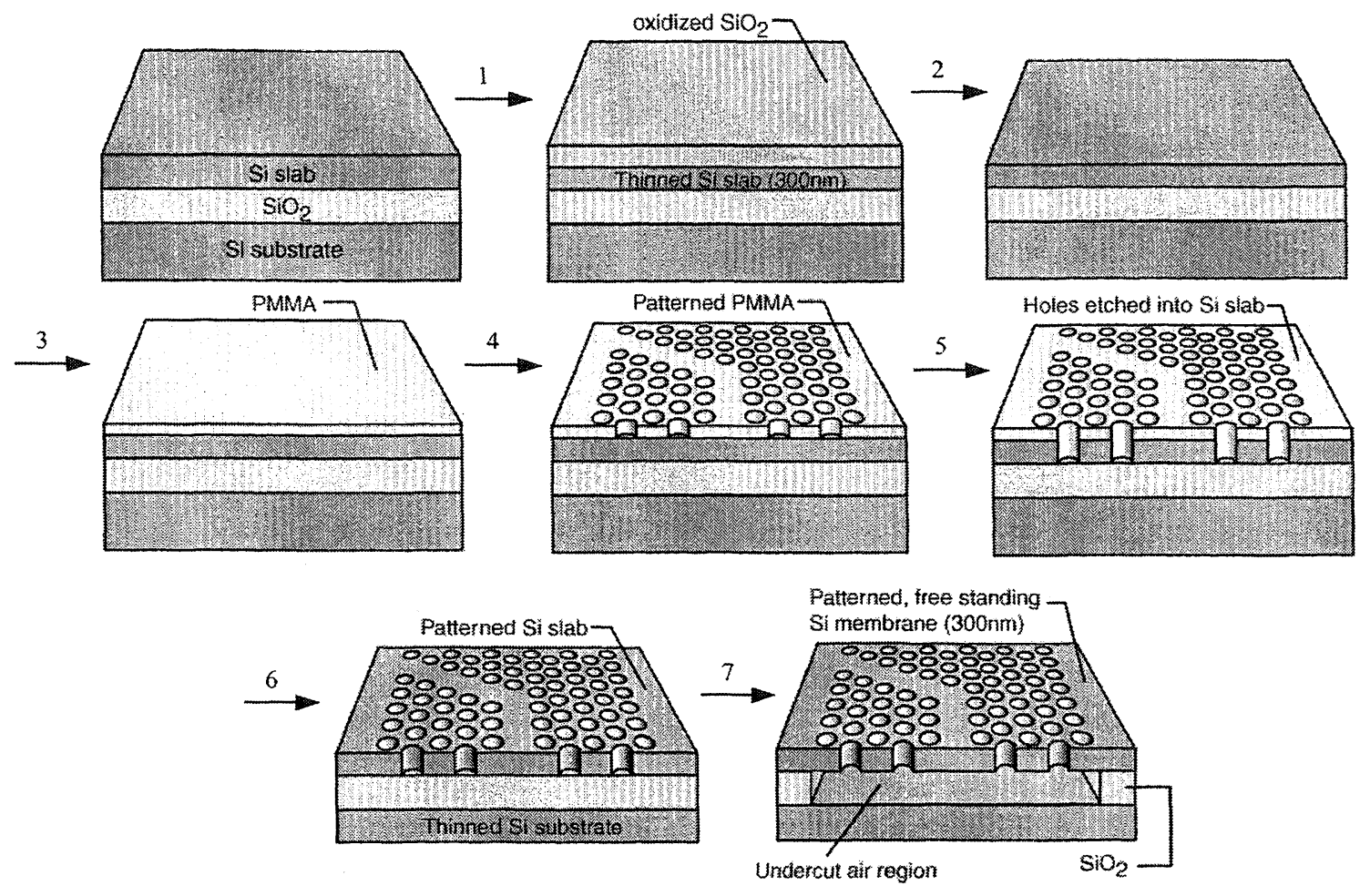

Figure 1. Fabrication Procedure: 1) oxidation and 2) HF dip in order to define the thickness of the Si slab; 3) deposition of PMMA and 4) electron-beam lithography to define patterns in PMMA; 5) CAIBE to transfer patterns into silicon; 6) removal of PMMA and thinning of the substrate; 7) $\mathrm{HF}$ dip to remove $\mathrm{SiO}_{2}$ layer underneath the waveguide in order to improve the vertical confinement of light.

The high selectivity and anisotropy of our fabrication process permit us to define high quality photonic crystal structures within SOI silicon wafers (Figure 2). Further details on the fabrication process can be found in previous publication ${ }^{8}$. We were able to define $500 \mu \mathrm{m}-1000 \mu \mathrm{m}$ long waveguides with different number of $60^{\circ}$ and $90^{\circ}$ bends. Due to the high-resolution e-beam lithography, we were able to achieve excellent uniformity of design parameters along the whole length of the waveguide.

In order to be able to couple the light in and out of the waveguide it is necessary to have access to both the input and the output facet of the guide. To do so, the samples were cleaved from both sides. Prior to cleaving, the sample was mechanically polished from the back side using $\mathrm{Al}_{2} \mathrm{O}_{3}$ polishing powder, and thinned from $500 \mu \mathrm{m}$ to below $100 \mu \mathrm{m}$. This thinning enabled us to obtain smooth cleaved edges at the both sides of the waveguide (Figure 2), which helps to reduce the insertion losses during optical coupling from a glass fiber to the photonic crystal waveguide. In order to increase the refractive index contrast in the vertical direction and thereby improve the vertical confinement of the light within the slab, the sacrificial $\mathrm{SiO}_{2}$ layer 
underneath the waveguides was removed by dissolution through the ion-etched holes using HF acid. This process leaves the Si waveguide and photonic crystal mirror membrane suspended in the air (Figure 2). The design parameters of fabricated photonic crystal waveguide are: interhole spacing (lattice constant) $\mathrm{a} \approx 530 \mathrm{~nm}$, hole radius $\mathrm{r} \approx 208 \mathrm{~nm}$, Si slab thickness $d \approx 300 \mathrm{~nm}$, in the case of hexagonal structure, and $a \approx 500 \mathrm{~nm}, r \approx 200 \mathrm{~nm}, \mathrm{~d} \approx 280 \mathrm{~nm}$, in the case of square structure.

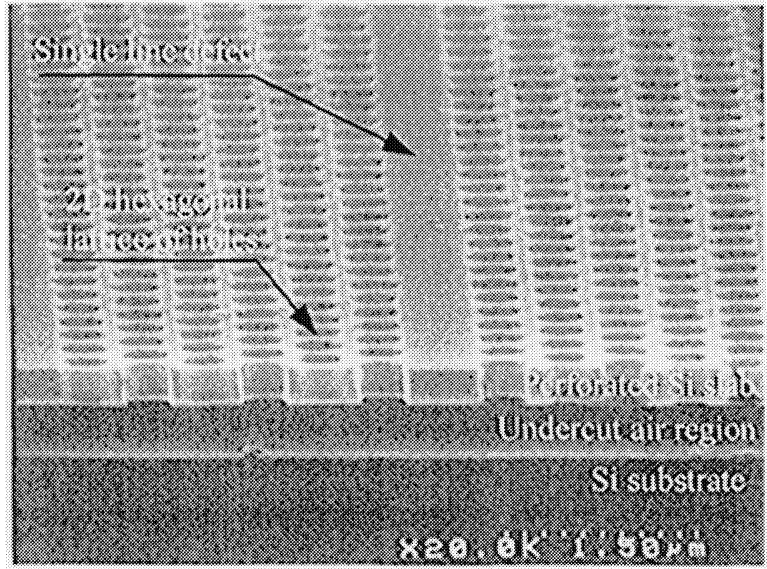

(a)

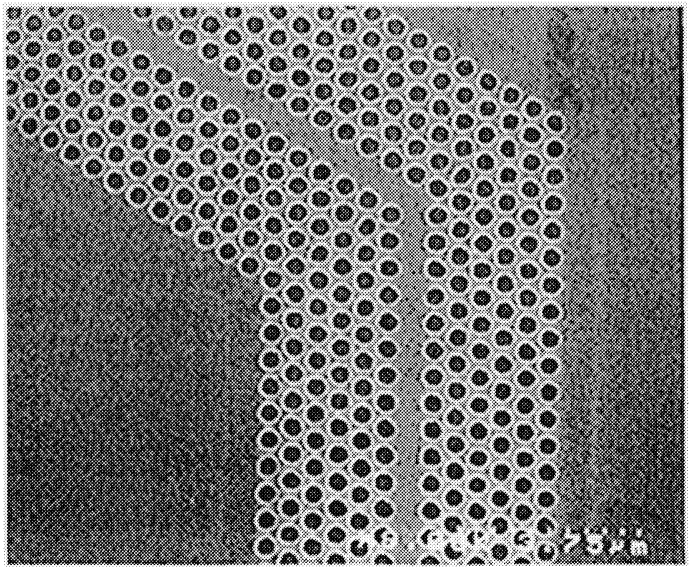

(b)

Figure 2. SEM micrograph of the fabricated waveguide of hexagonal symmetry.

a) Cross section of the fabricated structure. The waveguide is the line formed in the center by the absence of holes. The structure is suspended in the air in order to improve vertical confinement of light.

b) Top view of the $60^{\circ}$ bend in the hexagonal lattice. Undercut region extends for about $1 \mu \mathrm{m}$ around the waveguide.

An objective of this work was the characterization of guided modes transmitted through planar photonic crystal waveguides, and launched into the waveguides from an exterior tunable laser source. These measurements pose numerous challenges. First, it is necessary to couple light from a glass fiber into thin semiconductor slabs with reasonable efficiency. Second, the measured light intensity from the exit facet of the waveguide has to be effectively shielded from the scattered light coming from the input. Finally, we wish to determine the efficiency with which light is coupled into guided modes supported within the photonic crystal waveguide. Some of these challenges were addressed through careful design and fabrication, whereas others were addressed by choosing measurement of photonic crystal waveguides with bends, defined on thinned and cleaved silicon wafers, and by light output measurements both perpendicular and parallel to the wafer surface.

\section{CHARACTERIZATION}

A tunable semiconductor diode laser of rather modest power $(4 \mathrm{~mW}, 1440-1590 \mathrm{~nm})$ was used to characterize optical transmission. Butt-coupling of a single-mode fiber was used to introduce the laser output into the photonic crystal. Since fiber core diameter is around $10 \mu \mathrm{m}$, and slab thickness around $0.3 \mu \mathrm{m}$, coupling from the fiber to the waveguide was not efficient due to a large mode mismatch, and this problem remains to be solved. However, sufficient optical power $(\sim 10 \mu \mathrm{W})$ is coupled into the guide to carry out the required characterization of waveguiding within these structures. Waveguiding performance was observed by visualization of the guiding structure with two infrared television cameras. The first camera (\#1), positioned in the plane perpendicular to the sample, was used to observe the light scattered in the vertical direction. The second camera (\#2), positioned in the plane of the sample, was used to look at the cleaved output facet of the waveguide in order to observe the output (transmitted) signal. By careful manipulation of the input fiber, we were able to observe apparent guiding over two $90^{\circ}$ bends with waveguide segments of $\sim 200 \mu \mathrm{m}$ lengths (as shown in Figure 3) as well as guiding around a $60^{\circ}$ bend. Baba et al. have shown similar visualizations of light propagation in photonic crystals over shorter distances ${ }^{16}$. However, by reference to the output camera (\#2), we were able to confirm that true guiding by the photonic crystal is obtained by a different set of coupling conditions. Under these conditions, a clear guided mode is observed at the output facet of the waveguide, and very little light is scattered out of the waveguide perpendicular to the wafer. Whereas the image shown in Figure 3 is instrumental 
in qualitative visualization of light propagation within the photonic crystal structures, the optimization of true waveguiding requires the observation of the guided mode exiting from the photonic crystal sample. Since the waveguide lengths are on the order of 100 to 200 microns, preparation of a sample with high quality cleaved facets within these dimensions requires skill and patience.

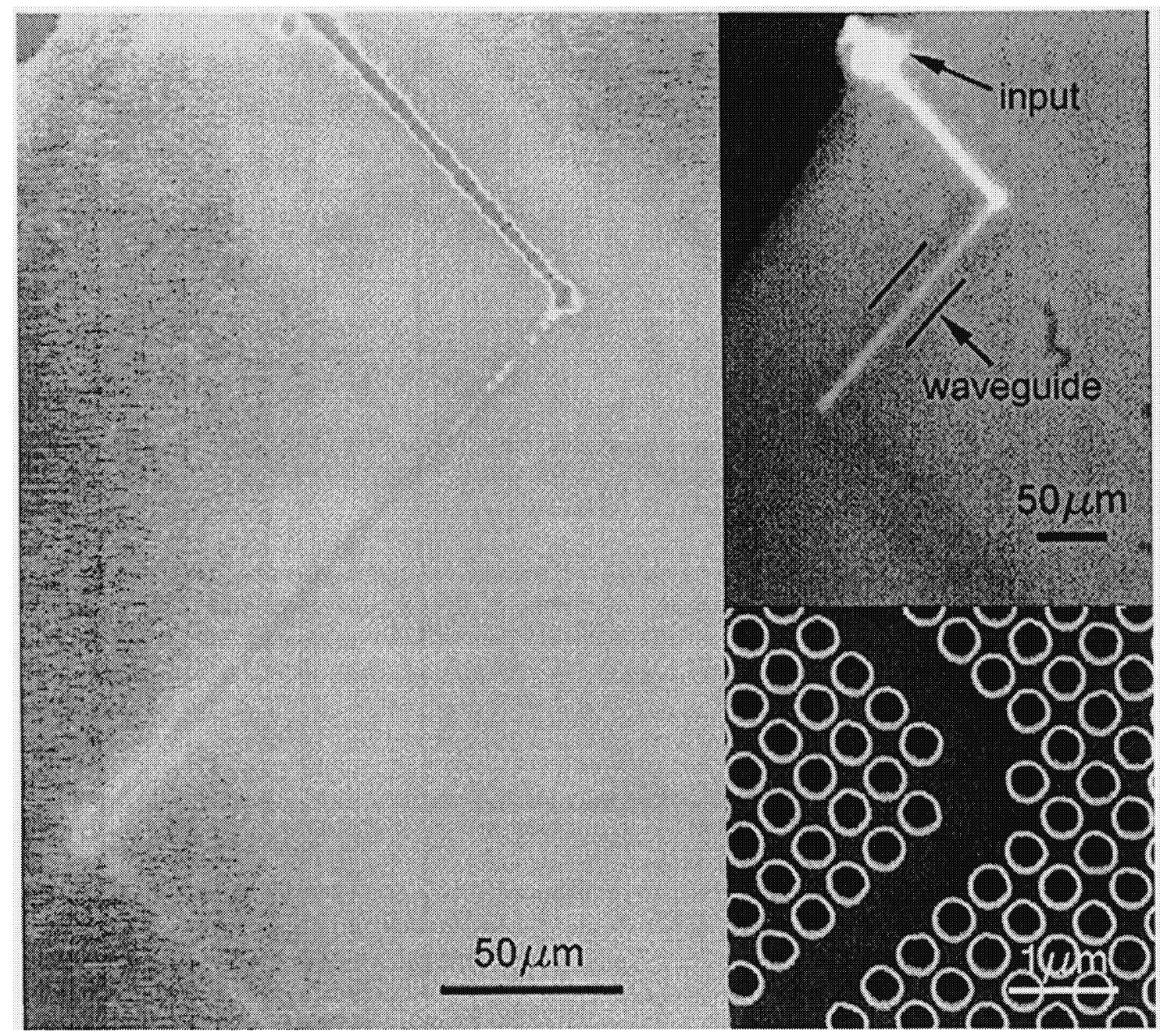

Figure 3. Light guided in the waveguide around two $90^{\circ}$ bends (top view). It can be seen that the light is confined to the waveguide (upper right inset). Inset in the lower right corner shows the SEM micrograph of the corner design in the square lattice.

For the case where the fiber was correctly aligned to the waveguide, (Figure 4a), camera \#2 detected two signals one mostly above the slab, coming directly from the input fiber (A in the Figure 4), and another, in the slab, representing the signal guided in the waveguide ( $\mathrm{B}$ in the Figure 4). The distance between the $60^{\circ}$ corner and the output of the waveguide was about $50 \mu \mathrm{m}$. This is sufficiently large to distinguish easily between these two signals. The Figure 4.c shows detected output power as a function of position along the cleaved output facet of the waveguide.

When fiber was not aligned to the waveguide (Figure $4 \mathrm{~b}$ ) camera $\# 2$ registered only one signal (A) - the one coming directly from the fiber. The other signal was not present since there was no light coupled into the waveguide. Similarly, when we moved the fiber in vertical direction guided signal (B) was present and not present depending on the relative position of the waveguide and the fiber. Therefore, we can conclude that signal B corresponds to the guided mode in this photonic crystal waveguide. This experimental result is persuasive evidence that control of light in photonic crystal circuits including tight corners can be achieved. 
Camera \#1

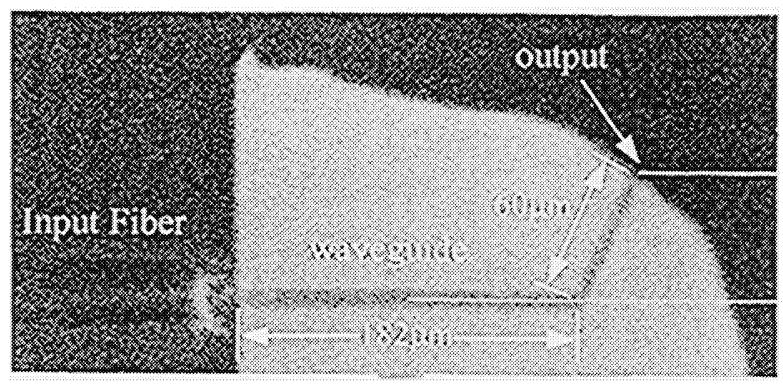

a)

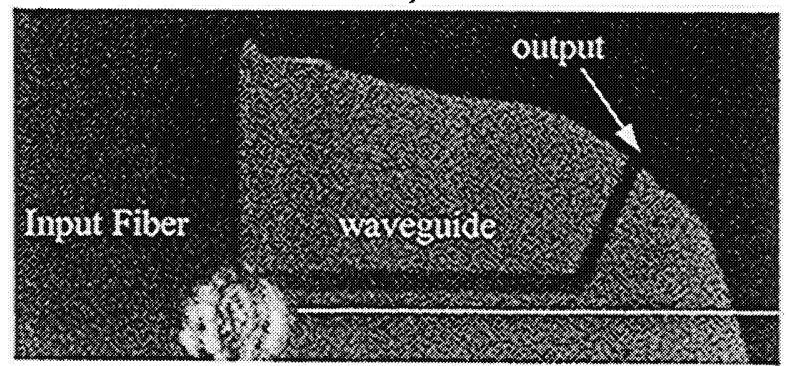

b)

\section{Camera \#2}

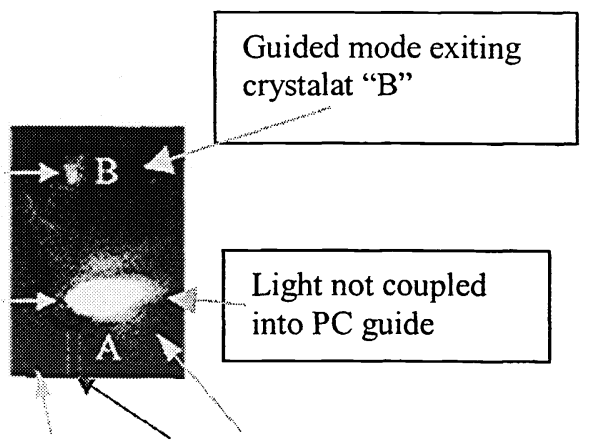

substrate slab air

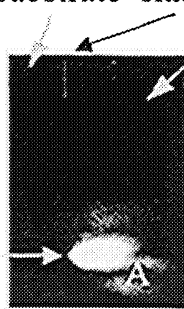

Figure 4. Waveguiding at $\lambda=1550 \mathrm{~nm}$ around a $60^{\circ}$ bend. Two cameras are used simultaneously, a top (camera \#1) and in-plane view (camera \#2) of the tested structure. When input fiber is aligned to the input of the waveguide (4.a), output signal, transmitted around $60^{\circ}$ bend is detected (signal B) and when it is misaligned (4.b) transmitted signal is not present. The position of the slab is indicated in the red color.

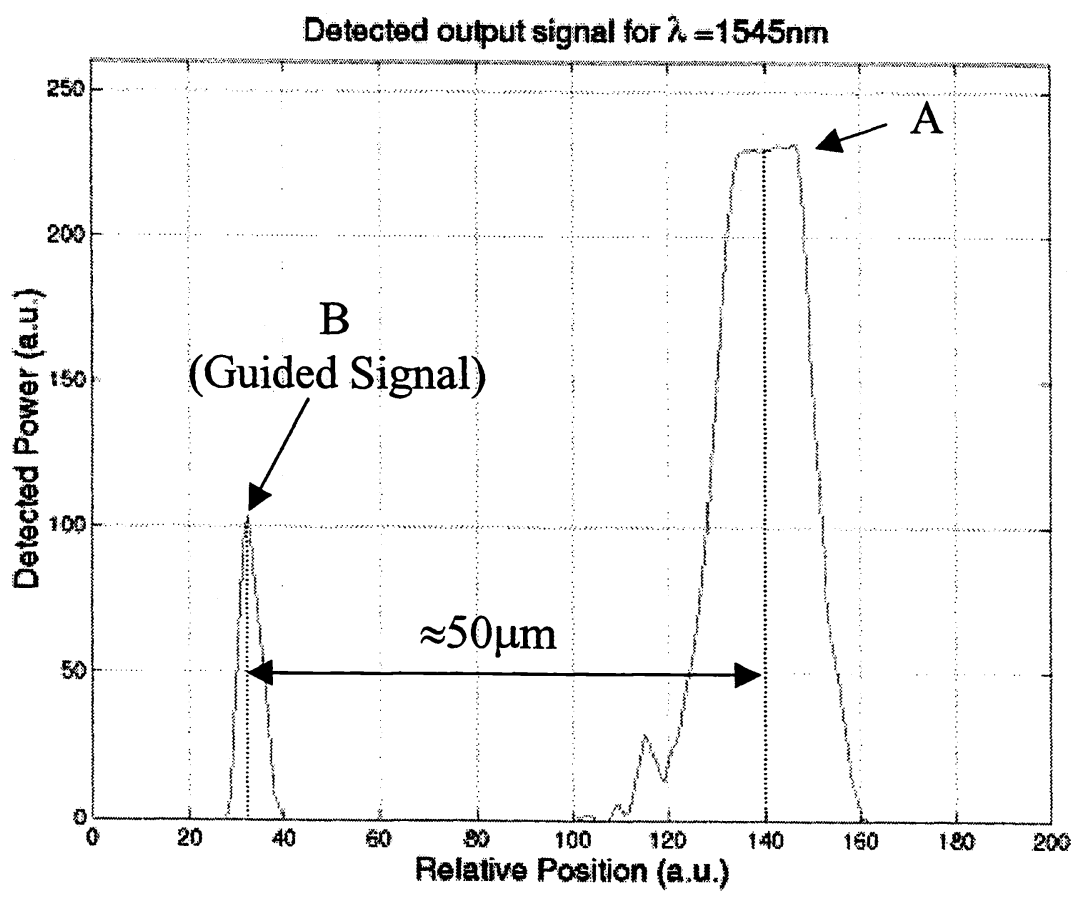

Figure 4.c. Detected output power (arbitrary units) at different positions along the cleaved output facet of the waveguide. Two peaks correspond to the signal coming directly from the input fiber (A), and the signal guided in the waveguide and transmitted around $60^{\circ}$ corner (B). The figure is obtained as a line-scan of the figure 4.a along the middle of the slab. 


\section{CONCLUSION}

We have demonstrated the fabrication and operation of planar photonic crystal waveguides. Three-dimensional analysis based on Maxwell's equations was used to find the suitable dimensions of the photonic crystal devices. While on one hand our research results represent encouraging progress toward the use of photonic crystal structures in planar photonic circuits, on the other they show that guiding conditions for real guides in three dimensions are more restrictive than previously thought using two-dimensional modeling. We have measured unambiguous guiding in these structures over the entire length of the sample. Under appropriate coupling conditions, this transmission appears to have tolerably low loss, a feature that is currently under study in our laboratory.

The authors gratefully acknowledge many fruitful technical discussions and collaborations with Oskar Painter, Reginald Lee and Professor Amnon Yariv.

\section{REFERENCES}

${ }^{1}$ J. D. Joannopoulos, R. D. Meade and J. N. Winn, Photonic Crystals ( Princeton University Press, Princeton, New Jersey, 1995)

${ }^{2}$ C. C. Cheng and A. Scherer, "Fabrication of photonic band-gap crystals" J. Vac. Sci. Technol. B 13, pp. 2696-2700, 1995

${ }^{3}$ S. Noda, N. Yamamoto, M. Imada, H. Kobayashi and M. Okano, "Alignment and stacking of semiconductor photonic bandgaps by wafer-fusion" J. Lightwave Tech 17, pp. 1948-1955, 1999

${ }^{4}$ S. Lin and J. G. Fleming, "A three-dimensional optical photonic crystal", J. Lightwave Tech. 17, pp. 1944-1947, 1999

${ }^{5}$ B. D'Urso, O. Painter, J. O'Brian, T. Tombrello, A. Yariv and A. Scherer, "Modal reflectivity in finite-depth twodimensional photonic-crystal microcavities", J. Opt. Soc. Am. B 15, pp. 1155-1159, 1998

${ }^{6}$ P. R. Villeneuve, S. Fan, S. G. Johnson, J. D. Joannopoulos, "Three-dimensional photon confinement in photonic crystals of low-dimensional periodicity" IEE Proc.-Optoelectron. 145, pp. 384-390, 1998

${ }^{7}$ O. Painter, R. K. Lee, A. Yariv, A. Scherer, J. D. O’Brien, P. D. Dapkus and I. Kim, "Two-dimensional photonic band-gap defect mode laser", Science 284, pp. 1819-1821, 1999

${ }^{8}$ M. Lončar., T. Doll, J. Vučković and A. Scherer, "Design and fabrication of silicon photonic crystal optical waveguides" Journal of Lightwave Technology 18, no. 10, pp. 1402-1411, 2000

${ }^{9}$ O. Painter, J. Vuckovic, A. Scherer, "Defect modes of a two-dimensional photonic crystal in an optically thin dielectric slab" J. Opt. Soc. Am. B 16, pp. 275-285, 1999

${ }^{10}$ I. El-Kady, M. M. Sigalas, R. Biswas, K. M. Ho, "Dielectric waveguides in two-dimensional photonic bandgap materials" J. Lightwave Tech., 17, pp. 2042-2049, 1999

${ }^{11}$ S.G. Johnson, S. Fan, P.R. Villeneuve, and J.D. Joannopoulos, "Guided modes in photonic crystal slabs" Physical Rev. B 60, pp. 5751-5758, 1999

${ }^{12}$ A. Mekis, J. C. Chen, I. Kurland, S. Fan, P. R. Villeneuve, J. D. Joannopoulos, "High transmission through sharp bends in photonic crystal waveguides" Physical Rev. Letters 77, pp. 3787-3790, 1996.

${ }^{13}$ S. Lin, E. Chow, V. Hietala, P. R. Villeneuve, J. D. Joannopoulos, "Experimental demonstration of guiding and bending of electromagnetic waves in a photonic crystal" Science 282, pp274-276, 1998

${ }^{14}$ S. Kuchinsky, D.C. Allan, N.F. Borelli, and J.C. Cotteverte, "3D localization in a channel waveguide in a photonic crystal with 2D periodicity" Optical Communications 175, pp 147-152, 2000

${ }^{15}$ M. Tokushima, H. Kosaka, A. Tomita, H. Yamada, "Lightwave propagation through a 120 degrees sharply bent single-linedefect photonic crystal waveguide" Appl. Phys. Letters 76, pp.952-954, 2000

${ }^{16} \mathrm{~T}$. Baba, N. Fukaya, J. Yonekura, "Observation of light propagation in photonic crystal optical waveguides with bends" Electronic Letters 35, pp. 654-655, 1999 